Research report

\title{
Remarkable complexity and variability of corticospinal tract defects in adult Semaphorin 6A knockout mice
}

\author{
Takuya Okada $^{\mathrm{a}}$, Kazuko Keino-Masu ${ }^{\mathrm{a}}$, Fumikazu Suto ${ }^{\mathrm{b}}$, Kevin J. Mitchell ${ }^{\mathrm{c}}$, Masayuki Masu ${ }^{\mathrm{a}, *}$ \\ ${ }^{a}$ Department of Molecular Neurobiology, Faculty of Medicine, University of Tsukuba, Tsukuba, Japan \\ ${ }^{\mathrm{b}}$ Department of Ultrastructural Research, National Institute of Neuroscience, National Center of Neurology and Psychiatry, Tokyo, Japan \\ ${ }^{\mathrm{c}}$ Smurfit Institute of Genetics and Institute of Neuroscience, Trinity College Dublin, Dublin, Ireland
}

\section{H I G H L I G H T S}

- Adult Sema6A knockout mice have corticospinal tract (CST) defects.

- The aberrant trajectories were evaluated by 3D reconstruction of $\mathrm{PKC} \gamma$-stained fibers.

- The CST defects in Sema6A knockout mice were highly complex and variable.

- 3D reconstruction is a powerful method to visualize these CST axon guidance defects.

\section{A R T I C L E I N F O}

\section{Keywords:}

Corticospinal tract

Axon guidance

Semaphorin $6 \mathrm{~A}$

Knockout mouse

3D reconstruction

\begin{abstract}
A B S T R A C T
The corticospinal tract (CST) has a complex and long trajectory that originates in the cerebral cortex and ends in the spinal cord. Semaphorin 6A (Sema6A), a member of the semaphorin family, is an important regulator of CST axon guidance. Previous studies have shown that postnatal Sema6A mutant mice have CST defects at the midbrain-hindbrain boundary and medulla. However, the routes the aberrant fibers take throughout the Sema6A mutant brain remain unknown. In this study, we performed 3D reconstruction of immunostained CST fibers to reevaluate the details of the abnormal CST trajectories in the brains of adult Sema6A mutant mice. Our results showed that the axon guidance defects reported in early postnatal mutants were consistently observed in adulthood. Those abnormal trajectories revealed by 3D analysis of brain sections were, however, more complex and variable than previously thought. In addition, 3D analysis allowed us to identify a few new patterns of aberrant projections. First, a subset of fibers that separated from and descended in parallel to the main bundle projected laterally at the caudal pons, subsequently changed direction by turning caudally, and extended to the medulla. Second, some abnormal fibers returned to the correct trajectory after deviating substantially from the original tract. Third, some fibers reached the pyramidal decussation normally but did not enter the dorsal funiculus. Section immunostaining combined with 3D reconstruction is a powerful method to track long projection fibers and to examine the entire nerve tracts of both normal and abnormal animals.
\end{abstract}

\section{Introduction}

Originating in the cerebral cortex and descending to the spinal cord, the corticospinal tract (CST) plays a role in controlling voluntary movement. Because of the length and complexity of its trajectory, a number of axon guidance molecules and their receptors have been implicated in steering the growing axons towards their proper targets during development (Canty and Murphy, 2008; Leyva-Diaz and LopezBendito, 2013). CST axons extend out of the cortical plate and direct towards the internal capsule at embryonic days 13-14 (E13-14). In these initial steps, Semaphorin 3A (Sema3A), Sema3C, and Netrin-1 guide the CST axons (Bagnard et al., 1998; Metin et al., 1997). At E1415 , the CST axons pass through the internal capsule and cerebral peduncle. Slit/Robo signaling is required for preventing the CST axons from entering the ventral forebrain and growing towards the midline (Bagri et al., 2002; Lopez-Bendito et al., 2007). After descending through the pons and medulla, the CST axons cross the midline at the pyramidal decussation and enter the dorsal funiculus at postnatal day 0 . Netrin-1,

Abbreviations: ABC, avidin-biotin complex; CST, corticospinal tract; DAB, 3,3'-diaminobenzidine; dlcst, dorsolateral corticospinal tract; DMSO, dimethyl sulfoxide; E, embryonic day; KO, knockout; PBS, phosphate-buffered saline; PKC $\gamma$, protein kinase C gamma; Sema, semaphorin; TBS, tris-buffered saline

* Corresponding author at: Department of Molecular Neurobiology, Faculty of Medicine, University of Tsukuba, 1-1-1 Tennodai, Tsukuba, Ibaraki 305-8575, Japan.

E-mail address: mmasu@md.tsukuba.ac.jp (M. Masu). 

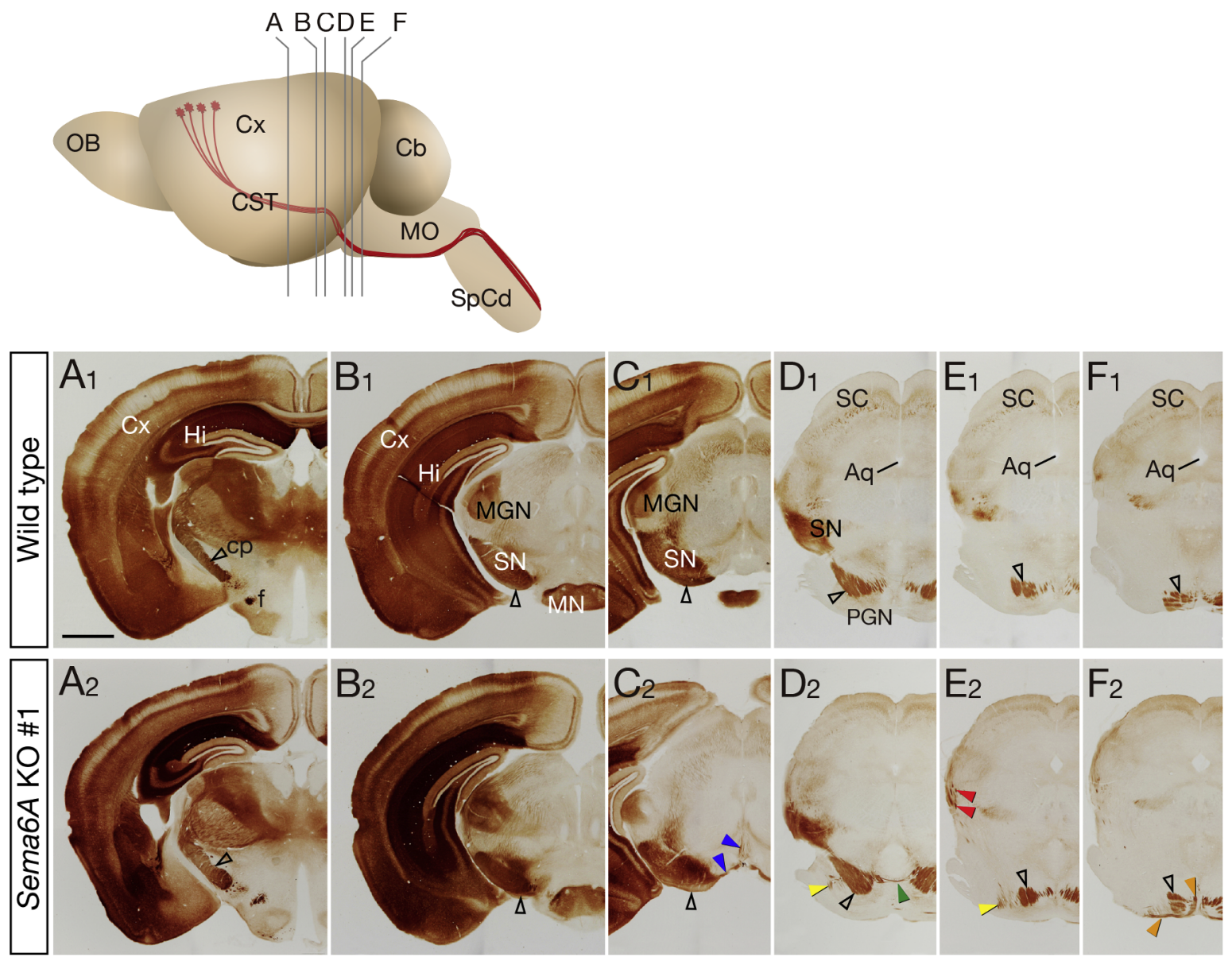

Fig. 1. CST defects at the midbrain-hindbrain boundary and in the pons of the Sema6A mutant brain. Immunohistochemical images of PKC $\gamma$ in adult wild-type $\left(\mathrm{A}_{1}-\mathrm{F}_{1}\right)$ and Sema6A KO $\left(\mathrm{A}_{2}-\mathrm{F}_{2}\right)$ brains. The coronal sections through the cerebral peduncle (cp) to the pons are shown. The positions of sections $(\mathrm{A})-(\mathrm{F})$ in the brain are shown in the upper panel. The open arrowheads indicate the normal projections of CST fibers. The filled arrowheads indicate misdirected fibers in the mutant brain; their colors correspond to those of the fibers in the schemes of Fig. 5B-B". Aq, aqueduct; Cb, cerebellum; Cx, cerebral cortex; f, fornix; Hi, hippocampus; MGN, medial geniculate nucleus; MN, mammillary nucleus; MO, medulla oblongata; OB, olfactory bulb; PGN, pontine gray nucleus; SC, superior colliculus; SN, substantia nigra; SpCd, spinal cord. The scale bar indicates $1.0 \mathrm{~mm}$.

L1, and NCAM are required to regulate the formation of the pyramidal decussation (Cohen et al., 1998; Finger et al., 2002; Rolf et al., 2002). In the spinal cord, Wnt signaling controls caudal growth of axons (Liu et al., 2005), and Ephrin-B3/EphA4 signaling prevents the CST axons from recrossing the midline (Coonan et al., 2001; Dottori et al., 1998; Kullander et al., 2001; Paixao et al., 2013).

The semaphorins are a large family of secreted and membrane-associated proteins that regulate nervous system development (Jongbloets and Pasterkamp, 2014; Kruger et al., 2005). They are classified into several groups on the basis of their structural characteristics: among vertebrate semaphorins, class 3 semaphorins are secreted proteins, whereas class 4-6 semaphorins are transmembrane proteins and class 7 semaphorins are glycophosphatidylinositol-anchored proteins. Semaphorin signals are mediated mainly by receptors of the plexin family. In addition to acting as ligands, transmembrane semaphorins including Sema6A also serve as receptors, mediating reverse signaling (Jongbloets and Pasterkamp, 2014; Kruger et al., 2005; Perez-Branguli et al., 2016).

During development, Sema6A plays a crucial role in guiding CST axons. In Sema6A knockout (KO) mice, several aberrant projections were observed in the pontine and medullary areas, whereas no obvious abnormalities were present rostral to the midbrain (Rünker et al., 2008). At the midbrain-hindbrain boundary, dorsal turning at the caudal end of the cerebral peduncle, abnormal midline crossing, and splitting into several bundles were observed (Rünker et al., 2008). At the level of the inferior olive, most fibers showed misrouting on the ventrolateral surface of the medulla (Faulkner et al., 2008; Rünker et al., 2008). Sema6A acts as a chemorepellent for axons that express its receptors Plexin-A2 (PlxnA2) and PlxnA4. Because Sema6A is highly expressed in the inferior olive, it was postulated that lateral expansion of the CST axons near the inferior olive in Sema6A KO mice was caused by the loss of Sema6A that constrains CST axons close to the midline through repulsion (Rünker et al., 2008). In addition, PlxnA2 and PlxnA4 are expressed in layer 5 cortical neurons, and the CST defects similar to those in Sema6A KO mice were observed in PlxnA4 KO mice, but not in PlxnA2 KO mice. It thus appears that Sema6A repels CST axons through PlxnA4 in this region (Faulkner et al., 2008; Rünker et al., 2008). This prominent defect in the caudal medulla was shown to be present in adulthood (Rünker et al., 2008), although analysis of other aberrant CST fibers in the adult brain has not been performed. In particular, because these previous studies were done using brain sections, the routes that the aberrant fibers take throughout the brain remain unknown. In addition, because neurodevelopmental abnormalities cause neurologic and psychiatric diseases (Rünker et al., 2011; Welniarz et al., 2016), phenotypic analysis of the adult brain is as important as that of the brain in its developmental stages.

In this study, we performed 3D reconstruction of the abnormal CST fibers in adult Sema6A mutant brains. This method enabled us to track the long and complex trajectory of the CST and to detect phenotypic variability among individual mutant mice. Our results clearly showed 

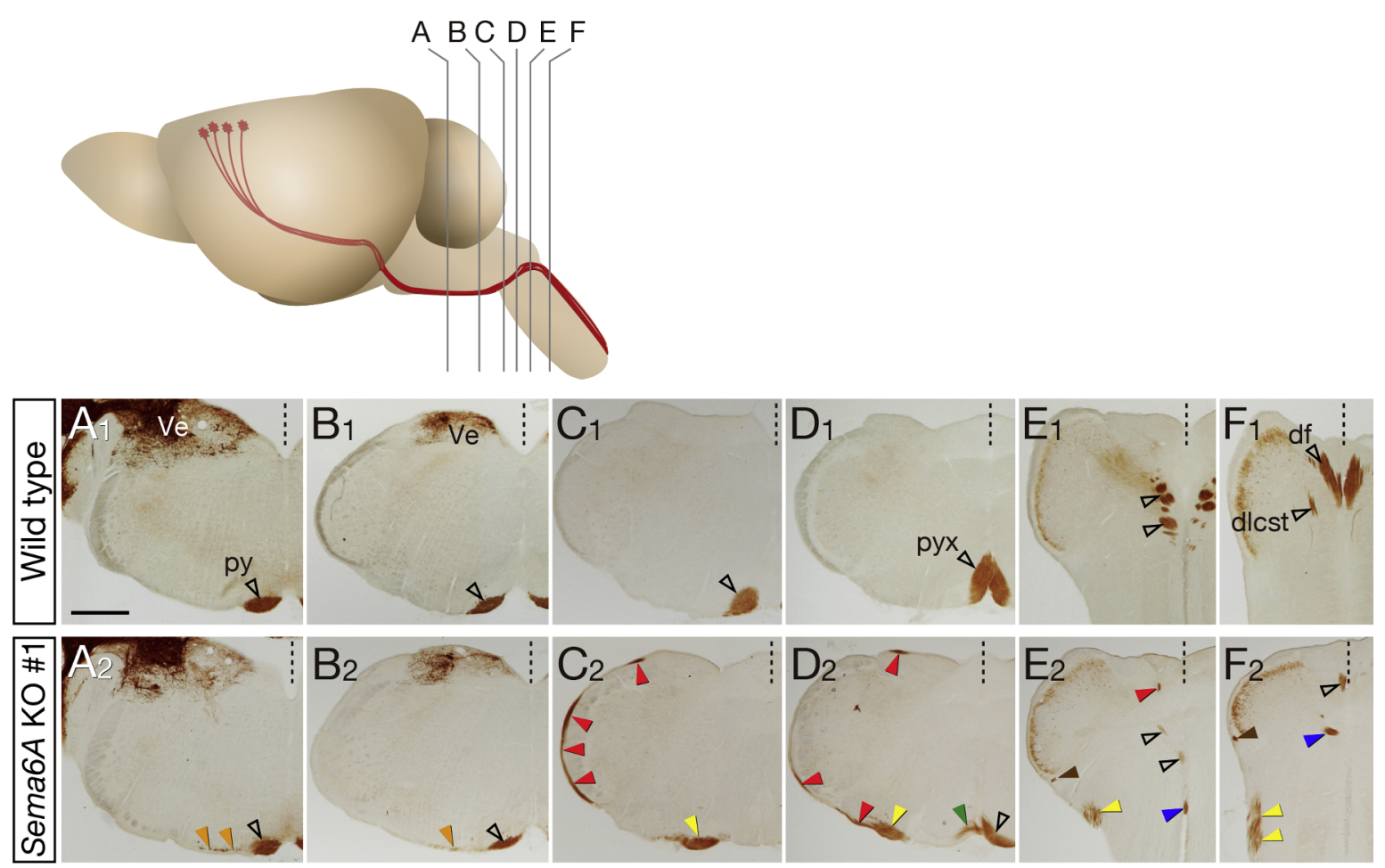

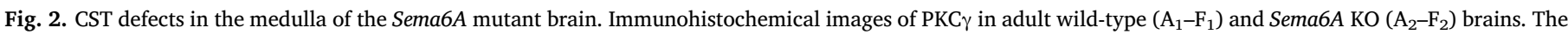

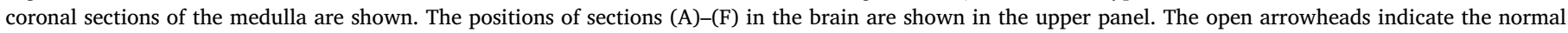

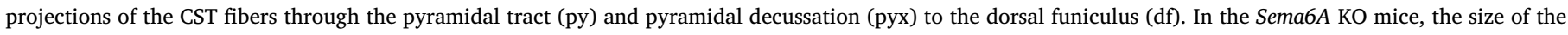

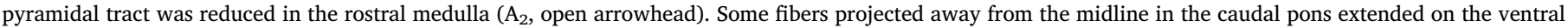

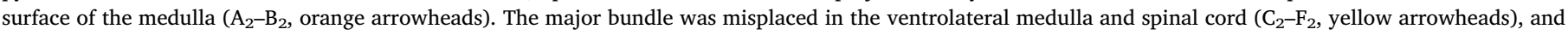

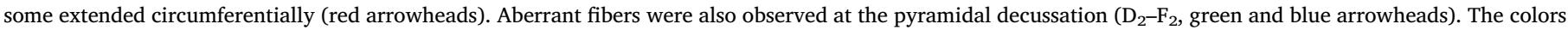

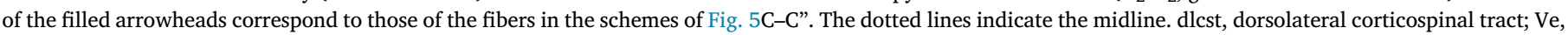
vestibular nucleus. The scale bar indicates $600 \mu \mathrm{m}$.

that the defects described in early postnatal mutants persist to adulthood. Moreover, we identified several patterns of CST defects that have not been previously described. Immunostaining combined with 3D reconstruction facilitates analysis of nerve tracts, especially long and complex projections such as the CST.

\section{Results}

\subsection{CST trajectory in the adult brain revealed by $P K C \gamma$ staining}

To reinvestigate the CST defects in adult Sema6A mutants, we first performed section immunostaining for protein kinase C gamma (PKC $\gamma$ ), a postnatal CST marker (Ding et al., 2005; Joshi et al., 2008; Mori et al., 1990). In the wild-type brain, PKC $\gamma$ was highly expressed in the cerebral cortex, hippocampus, thalamus, and cerebellum, whereas the staining was so scarce in the midbrain/hindbrain region that the PKC $\gamma$ positive CST fibers were easily tracked (Figs. 1 and 2). The CST fibers extended in the cerebral peduncle onto the ventrolateral surface of the diencephalon and passed immediately ventrolaterally to the substantia nigra, which was also strongly positive for PKC $\gamma$ (Fig. $1 B_{1}-C_{1}$ ). In the pons, the CST fibers extended ventrally and turned towards the midline (Fig. $1 D_{1}-F_{1}$ ). From the medulla to the spinal cord, the CST fibers were seen as a thick bundle in the pyramidal tract, pyramidal decussation, and dorsal funiculus (Fig. $2 \mathrm{~A}_{1}-\mathrm{F}_{1}$ ). In this region, in addition to the CST fibers, PKC $\gamma$ signals were observed in the vestibular nucleus and in the spinal and medullary dorsal horns (Fig. $2 \mathrm{~A}_{1}-\mathrm{F}_{1}$ ).

\subsection{CST defects in the midbrain-hindbrain junction of adult Sema6A KO mice}

In the Sema6A KO brain, the CST fibers appeared to be normal up to the caudal end of the cerebral peduncle (Fig. $1 \mathrm{~A}_{2}-\mathrm{B}_{2}$, open arrowheads), whereas a wide variety of aberrant projections were observed in the more caudal portion. We identified all the CST defects by tracking the aberrant fibers up to the cerebral peduncle in serial sections. Abnormal fibers with distinct characteristics are labelled in Figs. 1 and 2 with different colored arrowheads: other than orange, the same color in the 2 figures does not necessarily mean the same abnormality. At the level of the midbrain-hindbrain boundary, some fibers extended towards the midline and then turned dorsally (Fig. $1 \mathrm{C}_{2}$, blue arrowheads). At the level of the pontine gray nucleus, a small portion of fibers crossed the midline (Fig. $1 \mathrm{D}_{2}$, green arrowhead). A subset of misdirected fibers was observed immediately lateral to the cerebral peduncle (Fig. $1 D_{2}-E_{2}$, yellow arrowheads). In addition, abnormal fibers extended dorsally along the lateral surface of the midbrain (Fig. $1 \mathrm{E}_{2}$, red arrowheads). In the caudal pons, a fiber bundle located close to the midline projected laterally along the ventral brain surface (Fig. $1 \mathrm{~F}_{2}$, orange arrowheads). These defects are consistent with those in the early postnatal Sema6A KO mice described previously (Rünker et al., 2008). 
A

\section{D images}
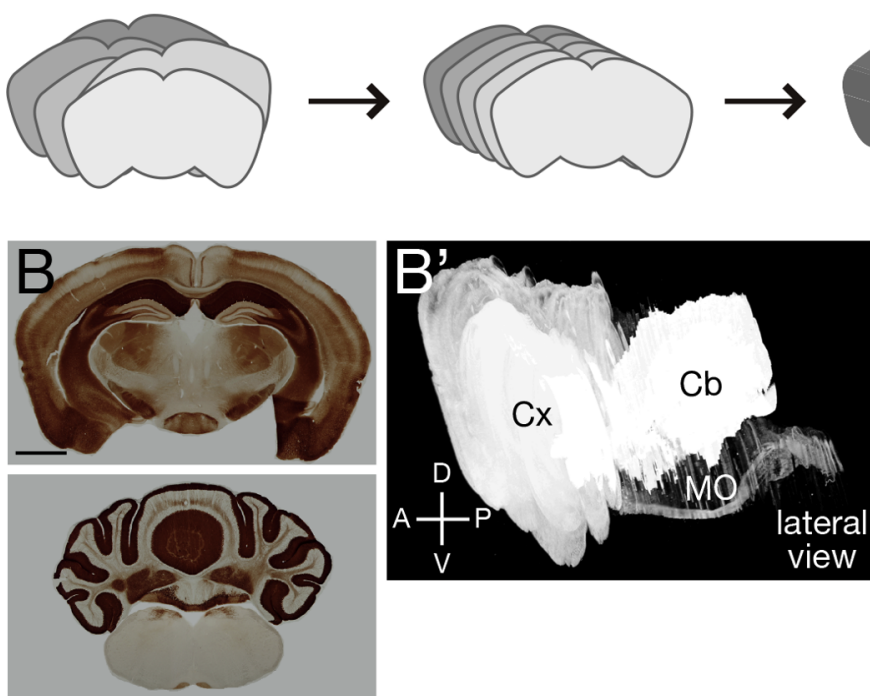

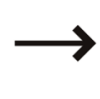

Alignment

3D reconstruction

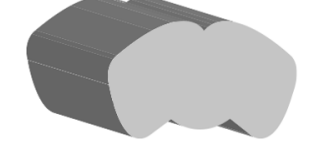

Fig. 3. 3D reconstruction of the CST. (A) Scheme of the $3 \mathrm{D}$ reconstruction. The 2D images of the serial sections were aligned on the basis of the shape of the sections and the location of the signals and reconstructed into a single $3 \mathrm{D}$ image. (B-B") $\mathrm{PKC} \gamma$ signals in the cerebral cortex, hippocampus, cerebellum, medulla, and spinal cord masked the CST in the whole brain preparation. (C-C") Processing of the original 2D images highlights the CST in the reconstructed $3 \mathrm{D}$ image. Anteroposterior (A-P) and dorsoventral (D-V) body axes are shown. Cb, cerebellum; cp, cerebral peduncle; $\mathrm{Cx}$, cerebral cortex; df, dorsal funiculus; MGN, medial geniculate nucleus; $\mathrm{MN}$, mammillary nucleus; MO, medulla oblongata; py, pyramidal tract; pyx, pyramidal decussation; SC, superior colliculus; SN, substantia nigra; Ve, vestibular nucleus. The scale bars indicate $1.3 \mathrm{~mm}$ (B'-B') and $1.0 \mathrm{~mm}$ (C'-C').
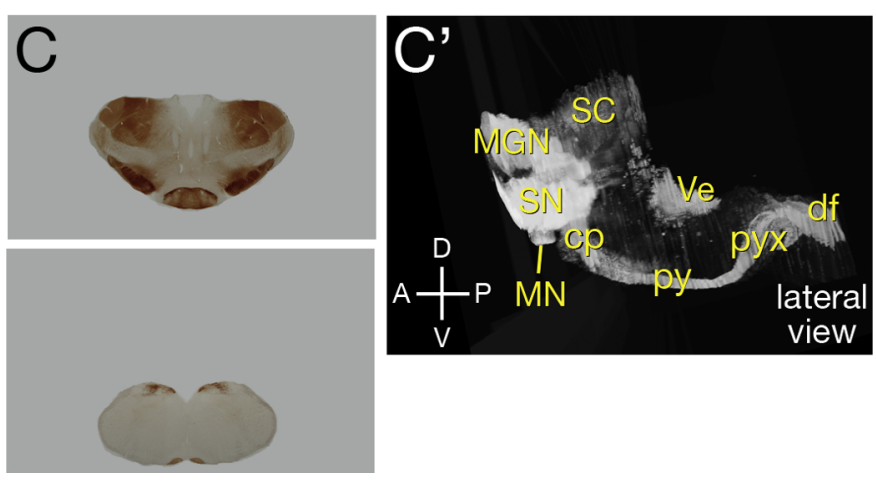

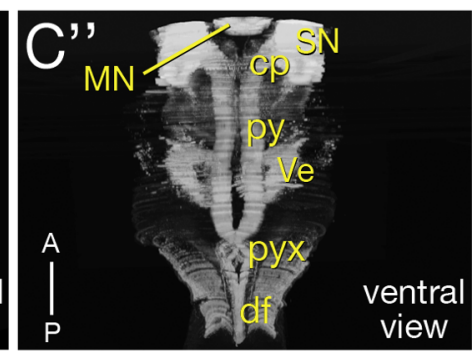

\subsection{CST defects in the caudal medulla of adult Sema6A KO mice}

Consistent with the gradual decrease in normal CST fibers at the midbrain-hindbrain boundary and pons, the size of the pyramidal tract was reduced in the rostral medulla of Sema6A KO mice (Fig. $2 \mathrm{~A}_{2}-\mathrm{B}_{2}$, open arrowheads). In the caudal medulla, the main fiber bundle deviated from the midline gradually and ran down the ventrolateral surface of the medulla (Fig. $2 \mathrm{C}_{2}-\mathrm{F}_{2}$, yellow arrowheads). This prominent defect was previously described in early postnatal Sema6A KO mice (Faulkner et al., 2008; Rünker et al., 2008). In addition, several abnormal fibers separated from the main bundle were identified. Misdirected fibers projected circumferentially in the dorsal direction (Fig. $2 \mathrm{C}_{2}-\mathrm{D}_{2}$, red arrowheads) and a small fiber bundle was observed near the lateral surface of the brain (Fig. $2 \mathrm{E}_{2}-\mathrm{F}_{2}$, brown arrowheads). In some cases, fibers reached the midline at the pyramidal decussation normally (Fig. $2 \mathrm{D}_{2}$, right side, open arrowhead), but many of them did not enter the dorsal funiculus after having crossed the midline successfully (Fig. $2 \mathrm{D}_{2}-\mathrm{F}_{2}$, green and blue arrowheads).

\subsection{D reconstruction of the CST trajectory}

To trace the CST fibers and obtain whole images of their trajectories, 3D images were reconstructed from the serial brain sections stained with anti-PKC $\gamma$ antibody. First, the 2D digital images of serial sections from the cerebral peduncle to the dorsal funiculus were aligned using AutoAligner software on the basis of the shape of the brain sections and the location of the signals. Then, stacks of the aligned images were processed into a single 3D image using Imaris software (Fig. 3A). When we observed the reconstructed 3D images, we found that strong $\mathrm{PKC} \gamma$ signals in the cerebral cortex, hippocampus, and cerebellum masked the CST trajectory embedded in these structures (Fig. 3B'-B'). Therefore, to see the CST fibers without disturbance, the signals in the thalamus, hypothalamus, brainstem, and spinal cord were extracted from the original 2D images (Fig. 3C), and the modified 2D images were realigned using the previously obtained information for optimal alignment. Finally, an undisturbed 3D image of the CST was successfully obtained (Fig. 3C'-C').

\subsection{D analysis of the normal CST trajectory in the wild-type brain}

In the wild-type brain, the CST fibers were seen as thick bundles in the ventral surface of the pons/medulla, pyramidal decussation, and dorsal funiculus of the spinal cord (Fig. 4, see movies 1-3 in Okada et al., 2019). At the midbrain-hindbrain boundary, the CST fibers turned medially, passed through the pons, and descended caudally on the ventral surface of the medulla. At the pons, the fibers located in the medial portion of the bundle were slightly defasciculated (Fig. 4B") and then refasciculated to form a thick bundle on the ventral medulla (Fig. 4C-C", py). At the pyramidal decussation (Fig. 4C-C", pyx), the CST fibers showed complicated trajectories. After dorsal turning and midline crossing, the fibers extended slightly laterally and split into several bundles on the contralateral side, and then the majority of fibers 
WT
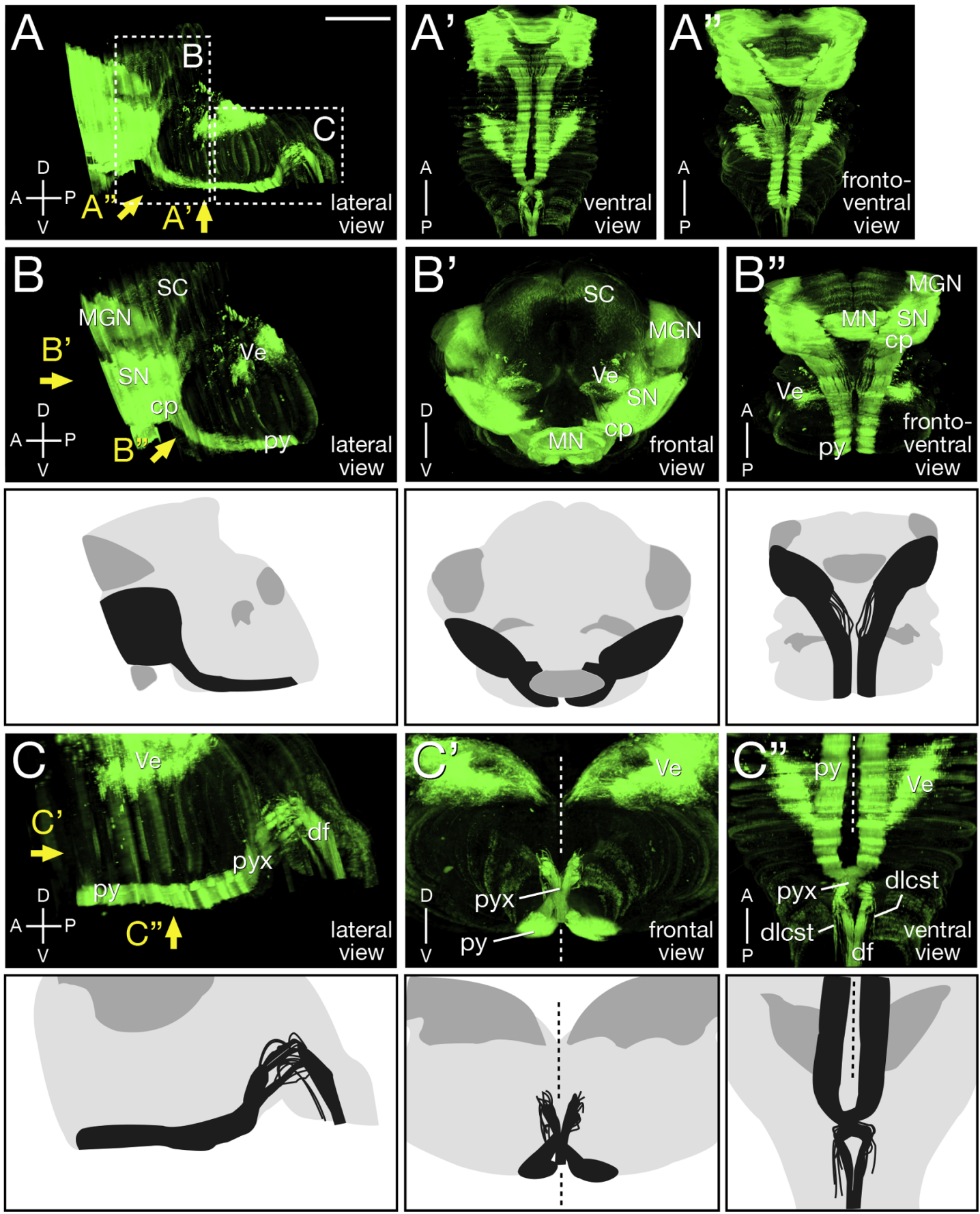

Fig. 4. 3D analysis of the wild-type CST. Representative 3D image of the wild-type CST. This sample is the same as that shown in Figs. 1 and 2 . The CST from the cerebral peduncle to the spinal cord (A-A"), from the cerebral peduncle to the rostral medulla (B-B"), and from the caudal medulla to the spinal cord (C-C") are shown. The arrows in (A), (B), and (C) indicate the direction of the view shown in pictures in (A'-A"), (B'-B'), and (C'-C"), respectively. In the lower panels in (B-B", C-C"), the substantia nigra and CST fibers are indicated in black, and the medial geniculate, mammillary, and vestibular nuclei, in dark gray. The CST fibers extended ventromedially from the midbrain towards the pons with slight defasciculation in the medial aspect of the thick bundle (B"). Then, the fibers refasciculated tightly to form a pyramidal tract (py) on the ventral surface of the medulla (A"-B"). (C-C") At the pyramidal decussation (pyx), after dorsal turning and midline crossing, most fibers entered the contralateral dorsal funiculus (df), whereas a small portion of fibers descended laterally to the dorsal funiculus as the dorsolateral corticospinal tract (dlcst). The dotted lines in (C'-C') indicate the midline. Anteroposterior (A-P) and dorsoventral (D-V) body axes are shown. cp, cerebral peduncle; MGN, medial geniculate nucleus, MN, mammillary nucleus; SC, superior colliculus; SN, substantia nigra; Ve, vestibular nucleus. The scale bars indicate $2.0 \mathrm{~mm}$ (A), $2.5 \mathrm{~mm}$ (A'-A"), $1.5 \mathrm{~mm}$ (B-B'), $1.85 \mathrm{~mm}$ (B”), $1.0 \mathrm{~mm}$ (C-C'), and $1.25 \mathrm{~mm}$ (C'). See movies 1-3 in Okada et al. (2019). 


\section{Sema6A KO \#1}
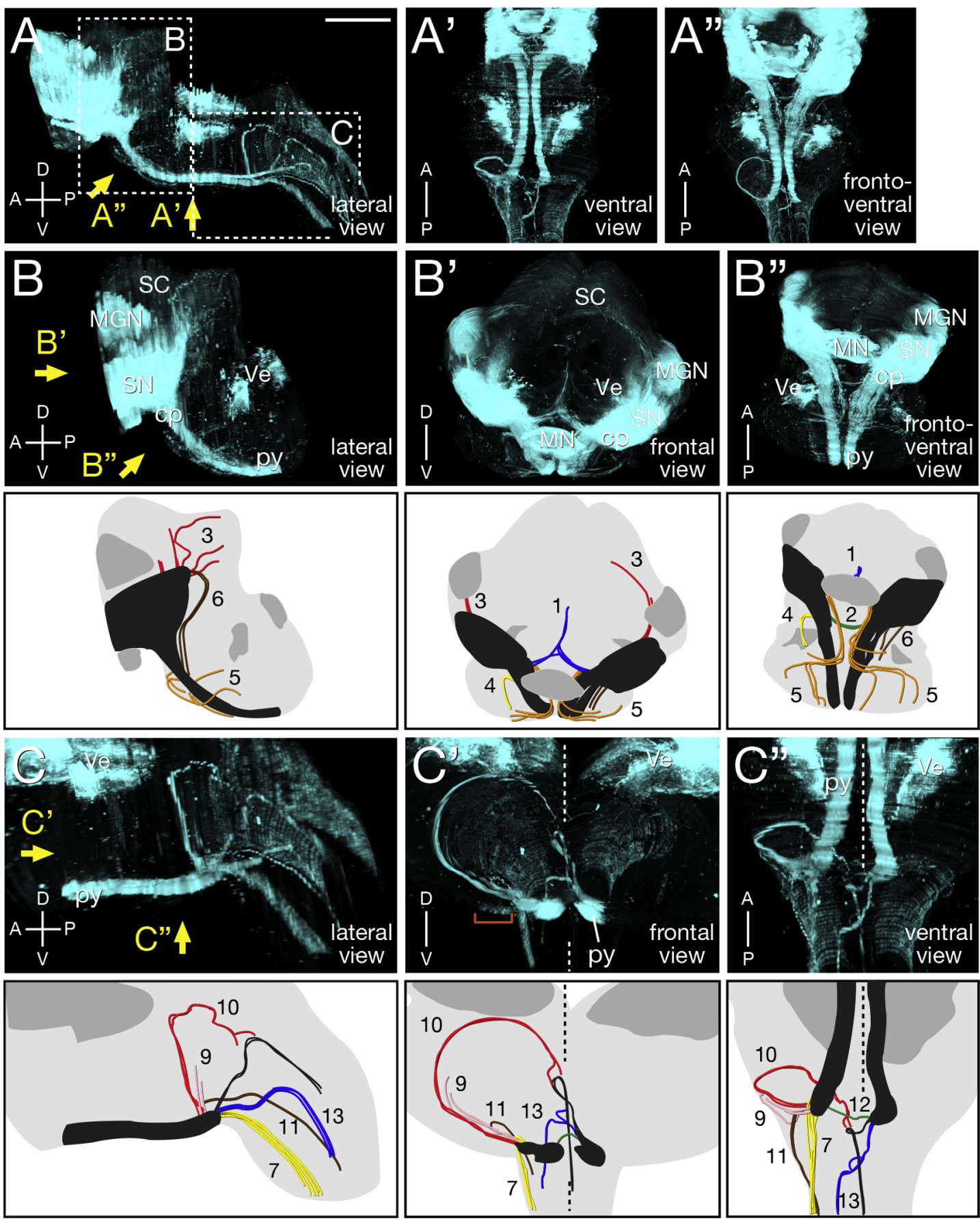

Fig. 5. CST defects in the Sema6A mutant brain (mouse \#1). 3D image of the CST of Sema6A KO mouse \#1. This sample is the same as that shown in Figs. 1 and 2. In the lower panels in (B-B", C-C"), the abnormal fibers are labeled with different colors and serial numbers. The substantia nigra and CST fibers are indicated in black, and the medial geniculate, mammilary, and vestibular nuclei, in dark gray. (B-B") In the midbrain, blue fibers (\#1) reached the ventral midline and turned dorsally. Red fibers (\#3) extended dorsally in the outermost lateral region of the midbrain, whereas brown fibers (\#6) returned to the pons (B-B"). In the pons, green fibers (\#2) crossed the midline. Yellow fibers (\#4) that projected laterally extended caudally. Orange fibers (\#5) passed across the main bundle and extended to the medulla (orange bracket in C'). (C-C') In the medulla, the main bundle in the right side of the brain deviated from the midline. Yellow (\#7) and brown (\#11) fibers extended on the ventrolateral surface and in the outermost lateral region of the spinal cord, respectively. Red (\#10) and pink (\#9) fibers extended circumferentially, and some of the red fibers entered the ipsilateral dorsal funiculus. In contrast, in the left side of the brain, the main bundle reached the pyramidal decussation and then split into 3 bundles. Although all the bundles crossed the midline, the black fibers entered the contralateral dorsal funiculus, whereas the blue (\#13) and green (\#12) fibers did not turn dorsally. Orange brackets indicate aberrant fibers (\#5) shown in B-B". Anteroposterior (A-P) and dorsoventral (D-V) body axes are shown. The scale bars indicate $2.0 \mathrm{~mm}$ (A), $2.5 \mathrm{~mm}$ (A'-A"), $1.5 \mathrm{~mm}$ (B-B'), $1.85 \mathrm{~mm}$ (B"), $1.0 \mathrm{~mm}$ (C-C'), and $1.25 \mathrm{~mm}$ (C"). See movies 4-6 in Okada et al. (2019). 


\section{Sema6A KO \#2}
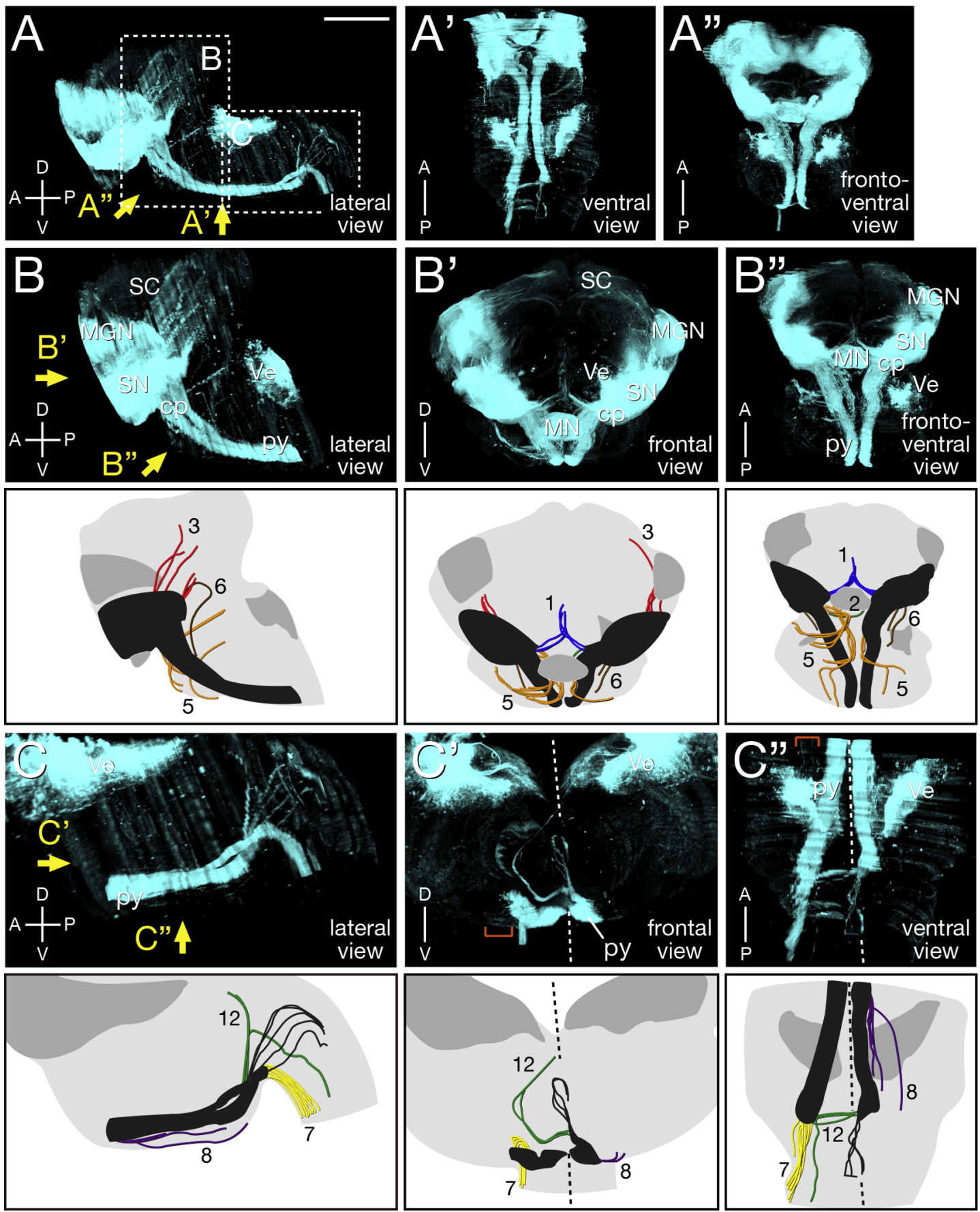

Fig. 6. CST axon guidance defects in the Sema6A mutant brain (mouse \#2). 3D image of the CST of Sema6A KO mouse \#2. (B-B") At the midbrain-hindbrain boundary and in the pons, the CST defects were similar to those observed in Sema6A KO mice \#1 and \#3. (C-C") In the caudal medulla, the defects appeared to be less severe than those observed in Sema6A KO mice \#1 and \#3. In the right side of the brain, almost all the fibers in the main bundle extended on the ventrolateral surface of the spinal cord. In the left side of the brain, most fibers, except for the deviated purple fibers (\#8), reached the pyramidal decussation, and then black fibers entered the contralateral dorsal funiculus. However, after crossing the midline, green fibers (\#12) extended away from the midline and then turned dorsally. The green fibers split, one extending towards the dorsal midline and the other caudally. The dotted lines in (C', C") indicate the midline. The orange brackets indicate the aberrant fibers (\#5) shown in B-B". Anteroposterior (A-P) and dorsoventral (D-V) body axes are shown. The scale bars indicate $2.0 \mathrm{~mm}$ (A), $2.5 \mathrm{~mm}$ (A'-A"), $1.5 \mathrm{~mm}$ (B-B'), $1.85 \mathrm{~mm}$ (B”), $1.0 \mathrm{~mm}$ (C-C'), and $1.25 \mathrm{~mm}$ (C'). See movies 7-9 in Okada et al. (2019). 


\section{Sema6A KO \#3}
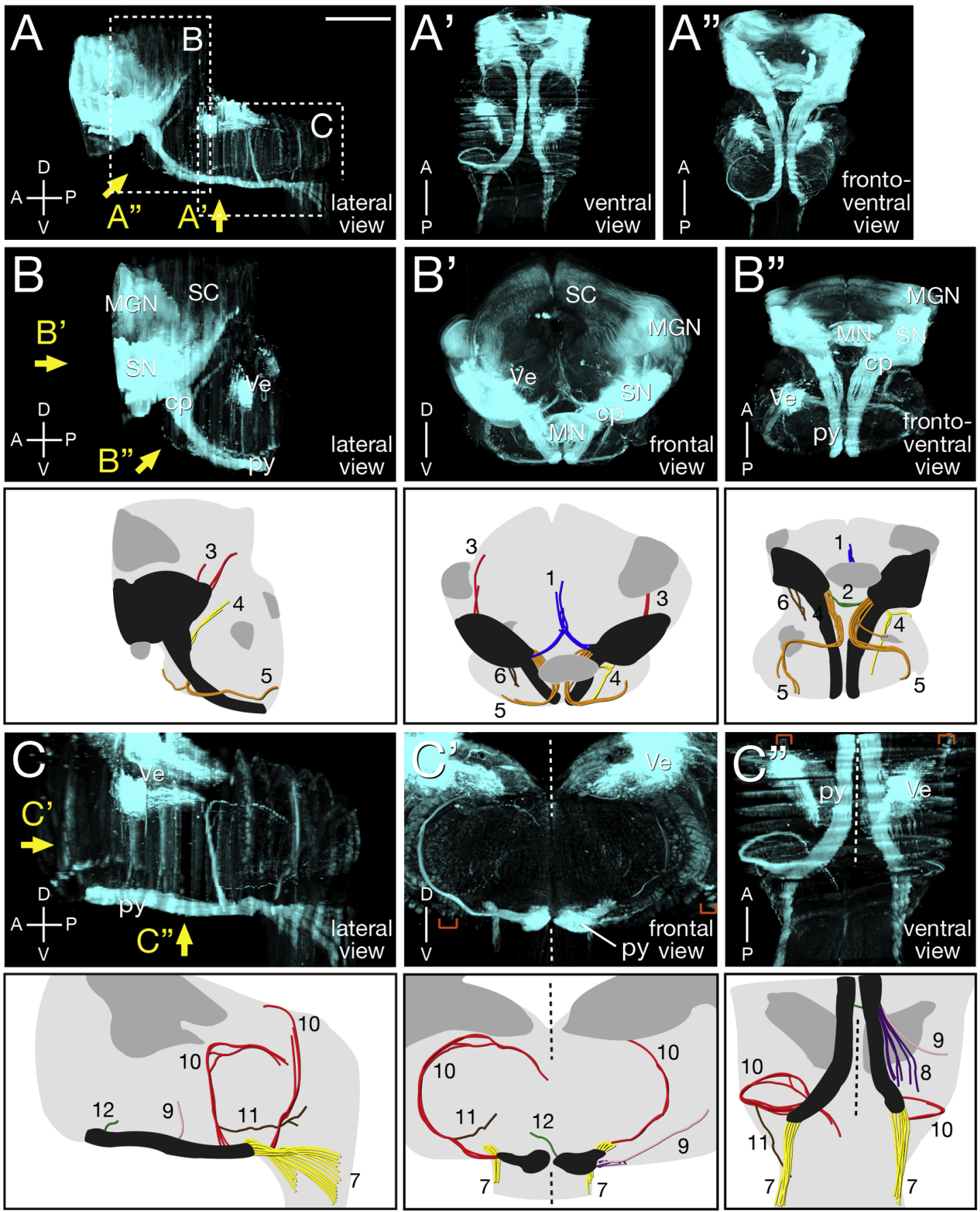

Fig. 7. CST axon guidance defects in the Sema6A mutant brain (mouse \#3). 3D image of the CST of Sema6A KO mouse \#3. (B-B") At the midbrain-hindbrain boundary and in the pons, the CST defects were similar to those observed in Sema6A KO mice \#1 and \#2. Yellow fibers (\#4) deviated laterally extended not only towards the caudal pons, but also towards the dorsal midbrain. (C-C") In the caudal medulla, the defects were somewhat symmetric. At the level of the facial nucleus, green fibers (\#12) crossed the midline. In addition to the laterally deviated purple fibers (\#8), pink fibers (\#9) extended circumferentially. The main bundles in both sides deviated from the midline, and yellow fibers (\#7) descended caudally, whereas red fibers (\#10) extended circumferentially towards the dorsal midline. In the right side of the brain, red fibers (\#10) entered the dorsal funiculus. Brown fibers (\#11) extended to the outermost lateral region of the spinal cord. The dotted lines in (C', C") indicate the midline. Anteroposterior (A-P) and dorsoventral (D-V) body axes are shown. The scale bars indicate $2.0 \mathrm{~mm}$ (A), $2.5 \mathrm{~mm}$ (A'-A"), $1.5 \mathrm{~mm}$ (B-B'), $1.85 \mathrm{~mm}$ (B'), $1.0 \mathrm{~mm}$ (C-C'), and $1.25 \mathrm{~mm}$ (C'). See movies 10-12 in Okada et al. (2019). 
refasciculated near the midline and entered the contralateral dorsal funiculus (Fig. 4C-C", df). The small portion of fibers that form the dorsolateral corticospinal tract (Steward et al., 2004) descended in a position lateral to the dorsal funiculus (Fig. 4C", dlcst). No misdirected fibers were observed along the entire trajectory in the wild-type brain.

\section{6. $3 D$ analysis of CST defects in the Sema6A KO brain}

In the Sema6A KO brains, many different types of abnormal CST fibers were observed (Figs. 5-7, see movies 4-12 in Okada et al., 2019). In the following description, the frequency of each type of CST defect is shown as the number of defects in all 8 tracts of the 4 Sema6A KO brains examined; the results of 3 representative mice are shown in Figs. 5-7. In the schematic diagrams of Figs. 5-7, the misdirected fibers with different characteristics are labeled with different colors and serial numbers. The color coding is used differently in the rostral (Figs. 5B-B", 6B-B", and 7B-B") and caudal (Figs. 5C-C", 6C-C", and 7C-C") regions. The serial numbers correspond to the types of defect (\#1 to \#13), which are described below.

\subsection{CST defects in the midbrain-hindbrain boundary of the Sema6A KO brain}

At the midbrain-hindbrain boundary (Figs. 5B-B", 6B-B", and 7B-B"; see movies 5, 8, and 11 in Okada et al., 2019), some fibers from both sides joined at the midline and then turned dorsally $(8 / 8$, blue fibers, \#1). In the pons, a small number of misdirected fibers crossed the midline (8/8, green fibers, \#2). Several small bundles ran in the dorsal direction in the lateral midbrain $(8 / 8$, red fibers, \#3). The fibers that projected laterally descended towards the caudal pons $(2 / 8$, yellow fibers, \#4). A significant number of fibers separated from the main bundle at its medial side, descended in parallel to the main bundle, passed across the main bundle on the surface of the pons, and projected laterally $(7 / 8$, orange fibers, \#5). In addition to these defects, which had previously been reported to be present in early postnatal Sema6A KO mice (Rünker et al., 2008), we found other defects that had not been previously described. A portion of the abnormal fibers in the lateral midbrain returned to the medulla $(5 / 8$, brown fibers, \#6). Aberrant fibers \#5 (indicated in orange in Figs. 5B-B", 6B-B", and 7B-B") continued to extend caudally on the ventral and lateral surfaces of the medulla (Figs. 5C', 6C'-C", and 7C'-C', orange brackets).

\subsection{CST defects in the medulla of the Sema6A KO brain}

In the caudal medulla (Figs. 5C-C", 6C-C", and 7C-C"; see movies 6,9 , and 12 in Okada et al., 2019), the main bundle projected away from the midline and descended along the ventrolateral surface of the brain to the ipsilateral spinal cord as described previously (Rünker et al., 2008; 6/8, yellow fibers, \#7). In addition, some CST defects were newly identified. At the level of the inferior olive, some fibers were defasciculated and positioned more laterally (2/8, purple fibers, \#8). Moreover, some fibers turned laterally and extended circumferentially in the dorsal direction (4/8, pink fibers, \#9; red fibers, \#10), and a portion of the misdirected fibers entered the dorsal funiculus $(2 / 8$, red fibers, \#10). A small portion of fibers also descended caudally along the outermost lateral spinal cord (2/8, brown fibers, \#11).

In 2 of the 8 tracts examined, the CST fibers appeared to reach the decussation point normally (Figs. 5C-C" and 6C-C", right side). However, instead of taking a normal route, these fibers split into several bundles near the decussation point; some of them entered the dorsal funiculus nearly normally (black fibers), whereas the others showed pathfinding errors. In 1 case, the misdirected fibers crossed the midline and extended laterally (Fig. 5C'-C', green fibers, \#12), whereas the others descended caudally (Fig. 5C-C", blue fibers, \#13). In another case, after crossing the midline, the aberrant fibers extended away from the midline and then turned dorsally (Fig. 6C-C", green fibers, \#12).
Taken together, more complex and variable defects than those described previously (Faulkner et al., 2008; Rünker et al., 2008) were newly identified.

\section{Discussion}

In this study, we performed 3D analysis of the CST trajectory to revisit the CST defects of Sema6A mutant mice. Our results clearly show that the CST defects persist into adulthood and are much more complex than was previously thought (Faulkner et al., 2008; Rünker et al., 2008). The defects at the midbrain-hindbrain boundary and in the pons are somewhat symmetric and similar among individuals, whereas those in the caudal medulla are highly variable. This may be related to the fact that proper formation of the pyramidal decussation is accomplished by the combined actions of Sema6A, Netrin-1, DCC, Unc5, L1, and NCAM, and thus when the route restriction of the growing CST axons by Sema6A is absent, different subsets of axons may respond to axon guidance molecules other than Sema6A in a distinct manner, leading to the wide variety of abberant projections. Intriguingly, some fibers (brown fibers \#6 and red fibers \#10) return to the correct trajectory after they deviate substantially from the original tract. In a previous study (Rünker et al., 2008), abnormal myelinated fibers were observed to be positioned circumferentially in the medulla, but whether they were derived from the CST was not established. In this study, we clearly showed that a subpopulation of the misdirected CST fibers (red fibers \#10) extends circumferentially towards the dorsal midline.

The most prominent defect is the ventrolateral projection of CST fibers in the region caudal to the inferior olive. It has been suggested that Sema6A expressed in the inferior olive is necessary for constraining PlxnA4-expressing CST axons close to the midline (Rünker et al., 2008). According to this model, in Sema6A mutant mice, the axons positioned far laterally do not decussate because they no longer respond to the midline guidance cues and those that remain near the midline cross the midline. Whether Sema6A has any effects on midline crossing and subsequent dorsal extension was not addressed (Rünker et al., 2008). In this study, we found several types of pathfinding errors in which the fibers came close to the midline at the decussation point but did not follow the normal trajectory (Figs. 5C-C" and 6C-C"). Most misdirected fibers crossed the midline but did not extend dorsally. Some fibers extended dorsally after the midline crossing but were displaced far laterally from the midline (Fig. 6C-C", green fibers, \#12). These results suggest the possible involvement of Sema6A in dorsal turning and/or extension of the CST axons at the pyramidal decussation.

In the wild-type brain, the CST fibers form a tight bundle throughout their trajectory, whereas they become once defasciculated and then refasciculated when they change direction at the level of the pons and pyramidal decussation. Multiple misdirected fibers in Sema6A mutant brains are frequently observed in these regions where the CST fibers are normally defasciculated in wild-type brains. It thus appears that these direction-changing positions are vulnerable to genetic perturbation that alters the coordination of multiple guidance molecules required for accurate direction change. Sema6A seems to play a role in bundling together the fibers that are defasciculated, although the molecular mechanisms remain to be elucidated. When the CST axons reach the pontine region, Sema6A is expressed in the axons. Moreover, because PlxnA4 mutants show no defects at this level, Sema6A may act as a receptor and may control the guidance of the defasciculated axons cell-autonomously (Faulkner et al., 2008; Rünker et al., 2008). In contrast, at the pyramidal decussation, PlxnA4 is expressed in the CST axons, whereas Sema6A is expressed in the surrounding regions, suggesting a non-cell-autonomous function of Sema6A as a ligand (Faulkner et al., 2008; Rünker et al., 2008). Therefore, future studies are required to determine the mode of Sema6A signaling in CST axon guidance. In addition, given that CST malformations-in particular, altered midline crossing at the pyramidal decussation or in the spinal cord-lead to human CST developmental disorders (Welniarz et al., 
2016), it is intriguing to examine whether Sema6A KO mice show behavioral abnormalities of voluntary movements.

Recently, new methods of 3D analysis of neuronal and nonneuronal tissues have been developed (Susaki et al., 2014). A combination of whole-mount immunostaining, tissue clearing, and light sheet microscopy is useful to visualize neuronal connections, activity, proliferation, and degeneration (Belle et al., 2014; Hama et al., 2011; Renier et al., 2014). To obtain clear 3D images, screening of antibodies, optimization of the immunostaining protocol, selection of the appropriate clearing protocol, and establishment of imaging and processing setups are critical. In contrast, 3D analysis using a combination of section immunostaining and 3D reconstruction requires fewer experimental considerations. In particular, because antibodies penetrate into sections more easily and rapidly than they do in whole-mount preparations, standard immunostainig protocols can be used. In addition, it is advantageous that high-resolution images of immunostained sections are available simultaneously. However, because the depth information of the signals in the section is lost, this method may not be appropriate when quantitative analysis along the z-axis is required. Three-dimensional reconstruction with electron microscopy has been developed for high-resolution imaging (Helmstaedter et al., 2013), but it is not practical to apply this method to acquire a whole image of large-sized specimens. The method of 3D analysis used in this study is a powerful option to visualize axon guidance defects along the entire length of the trajectory.

Finally, the results presented here provide a dramatic illustration of an important general point-that neurodevelopmental processes are essentially probabilistic (Mitchell, 2007). In wild-type brains, this fact is obscured by the overall robustness of the developmental program, which generates a wild-type outcome with nearly $100 \%$ probability. But when genes encoding neurodevelopmental information are mutated, the typical outcome is not just a shift from the wild-type to a specific mutant phenotype, but also an increase in variability. From this perspective, we can see that incomplete penetrance of particular anatomic defects reflects variation that is inherent to the developing organism and not caused by any external factors. This may be an important third source of variance in neuroanatomic and behavioral traits in humans, in addition to genetic differences and different family environments. Furthermore, while a certain risk of neurologic or psychiatric disease may be inherited owing to mutations affecting neural development (Hussman et al., 2011; Rünker et al., 2011), the actual outcome in any individual will also be affected by probabilistic processes of the type illustrated here (Mitchell, 2015).

\section{Experimental procedure}

\subsection{Knockout mice}

The Sema6A KO mice were described previously (Rünker et al., 2008). All animal experiments were approved by and performed according to the guidelines of the Animal Care and Use Committees of the University of Tsukuba and the National Institute of Neuroscience, National Center of Neurology and Psychiatry.

\subsection{Immunohistochemistry}

Adult mice were transcardially perfused with $4 \%$ paraformaldehyde in phosphate-buffered saline (PBS) under deep anesthesia with diethyl ether. After the brains had been removed, they were immersed in the same fixative at $4{ }^{\circ} \mathrm{C}$ overnight. The fixed brains were then incubated in $30 \%$ sucrose/PBS at $4{ }^{\circ} \mathrm{C}$ overnight for cryoprotection and embedded in OCT compound (Sakura Finetek Japan, Tokyo, Japan). For PKC $\gamma$ immunostaining, 50- $\mu$ m-thick cryosections were sequentially collected into 1 well of a 6-well dish containing PBS. Each well thus contained the sections at intervals of $300 \mu \mathrm{m}$, and the brain sections were easily arranged on glass slides in the correct order after staining. The sections were washed with PBS several times to remove the OCT compound. The sections were dehydrated in serial dilutions of methanol from $25 \%$ to $80 \%$ in PBS with $0.1 \%$ Tween-20 (PBT) and then washed with $80 \%$ methanol, 20\% dimethyl sulfoxide (DMSO) for $5 \mathrm{~min}$. For inactivation of the endogenous peroxidase activity, the sections were incubated in $3 \% \mathrm{H}_{2} \mathrm{O}_{2}$ in $80 \%$ methanol, $20 \%$ DMSO for $30 \mathrm{~min}$. After being washed with $80 \%$ methanol, $20 \%$ DMSO for $5 \mathrm{~min}$, the sections were rehydrated in serial dilutions of methanol from $80 \%$ to $25 \%$ in PBT and then washed with PBT for $5 \mathrm{~min}$. The sections were incubated twice overnight with anti-PKC $\gamma$ antibody (1:200; Frontier Institute, Hokkaido, Japan) in $0.5 \%$ blocking reagent (Roche Diagnostics, Mannheim, Germany) in PBT at $4{ }^{\circ} \mathrm{C}$. The sections were washed with PBT for $15 \mathrm{~min}$ 6 times and then incubated with biotin-conjugated anti-rabbit IgG antibody (1:600; Vector Laboratories, Burlingame, CA, USA) in $0.5 \%$ blocking reagent in PBT for $2 \mathrm{~h}$. After being washed with PBT for 15 min 5 times and with Tris- $\mathrm{HCl}, \mathrm{pH} 7.6$, containing $0.8 \% \mathrm{NaCl}, 0.02 \%$ $\mathrm{KCl}$, and $0.1 \%$ Tween-20 (0.1\% Tween-20/TBS) for $15 \mathrm{~min}$, the sections were incubated with avidin-biotin complex (Vectastain Elite ABC kit; Vector Laboratories) for $30 \mathrm{~min}$. The sections were washed with $1 \%$ Tween-20/TBS for $20 \mathrm{~min}$, then with $0.1 \%$ Tween-20/TBS for $20 \mathrm{~min}$ twice, and thereafter kept in the same solution at $4{ }^{\circ} \mathrm{C}$ overnight. The sections were incubated with 3,3'-diaminobenzidine (DAB; Vector Laboratories) for $10 \mathrm{~min}$. All steps were performed at room temperature unless otherwise indicated. After staining, the sections were mounted on MAS-coated glass slides (Matsunami Glass Industries, Osaka, Japan) and air-dried overnight. The coverslips were mounted using Fluoromount-G (SouthernBiotech, Birmingham, AL, USA). Bright field images were obtained using a fluorescence microscope (BZ-8000; Keyence, Osaka, Japan).

\section{3. $3 D$ reconstruction}

The 2D images of serial sections stained with PKC $\gamma$ antibody were aligned using AutoAligner alignment software (Bitplane, Zürich, Switzerland) on the basis of the shape of the sections and the location of the signals. The alignment information (X-, Y-, and Z-coordinates and the angle of rotation) was saved for subsequent analysis. Stacks of the aligned images were imported into Imaris software (Bitplane) and transformed into 3D images. Because strong PKC $\gamma$ signals in the cerebral cortex, hippocampus, and cerebellum masked the CST in a $3 \mathrm{D}$ image (Fig. 3B'-B'), the signals in the thalamus, hypothalamus, brainstem, and spinal cord were extracted from the original 2D images (Fig. 3C) using Photoshop software (Adobe Systems, San Jose, CA, USA). The saved alignment information was applied to the modified 2D images to reconstruct 3D images of the CST. Pseudocolors of pale green and blue were assigned to the wild-type and mutant CSTs, respectively.

\section{Author contributions}

T.O., K.K.-M., and M.M. designed the research and performed the experiments. T.O. carried out the image analysis and 3D reconstruction. F.S. prepared the fixed mouse brains. K.J.M. generated the Sema6A knockout mice. T.O., K.K.-M., K.J.M., and M.M. wrote the manuscript. All the authors read and approved the final manuscript.

\section{Funding}

This work was supported by Kakenhi grants (grant numbers 22123006, 25293065) from the Japanese Ministry of Education, Culture, Sports, Science, and Technology (MEXT) and from the Japan Society for the Promotion of Science (JSPS) to M.M.

\section{Competing interests}

The authors have no competing interests to declare. 


\section{Acknowledgements}

We thank T. Shiga, H. Ichijo, T. Masuda, and F. Miyamasu for critical reading of the mansucript and $\mathrm{H}$. Yuyama, S. Imaizumi, and Y. Iwahashi for their experimental support.

\section{References}

Bagnard, D., Lohrum, M., Uziel, D., Puschel, A.W., Bolz, J., 1998. Semaphorins act as attractive and repulsive guidance signals during the development of cortical projections. Development 125, 5043-5053.

Bagri, A., Marin, O., Plump, A.S., Mak, J., Pleasure, S.J., Rubenstein, J.L., Tessier-Lavigne, M., 2002. Slit proteins prevent midline crossing and determine the dorsoventral position of major axonal pathways in the mammalian forebrain. Neuron 33 (2), 233-248.

Belle, M., Godefroy, D., Dominici, C., Heitz-Marchaland, C., Zelina, P., Hellal, F., Bradke, F., Chedotal, A., 2014. A simple method for 3D analysis of immunolabeled axonal tracts in a transparent nervous system. Cell Rep. 9 (4), 1191-1201.

Canty, A.J., Murphy, M., 2008. Molecular mechanisms of axon guidance in the developing corticospinal tract. Progr. Neurobiol. 85 (2), 214-235.

Cohen, N.R., Taylor, J.S., Scott, L.B., Guillery, R.W., Soriano, P., Furley, A.J., 1998. Errors in corticospinal axon guidance in mice lacking the neural cell adhesion molecule L1. Curr. Biol. 8 (1), 26-33.

Coonan, J.R., Greferath, U., Messenger, J., Hartley, L., Murphy, M., Boyd, A.W., Dottori, M., Galea, M.P., Bartlett, P.F., 2001. Development and reorganization of corticospinal projections in EphA4 deficient mice. J. Comp. Neurol. 436 (2), 248-262.

Ding, Y.Q., Xiang, C.X., Chen, Z.F., 2005. Generation and characterization of the PKC gamma-Cre mouse line. Genesis 43 (1), 28-33.

Dottori, M., Hartley, L., Galea, M., Paxinos, G., Polizzotto, M., Kilpatrick, T., Bartlett, P.F., Murphy, M., Kontgen, F., Boyd, A.W., 1998. EphA4 (Sek1) receptor tyrosine kinase is required for the development of the corticospinal tract. PNAS 95 (22), 13248-13253.

Faulkner, R.L., Low, L.K., Liu, X.B., Coble, J., Jones, E.G., Cheng, H.J., 2008. Dorsal turning of motor corticospinal axons at the pyramidal decussation requires plexin signaling. Neural Dev. 3, 21.

Finger, J.H., Bronson, R.T., Harris, B., Johnson, K., Przyborski, S.A., Ackerman, S.L., 2002. The netrin 1 receptors Unc5h3 and Dcc are necessary at multiple choice points for the guidance of corticospinal tract axons. J. Neurosci. 22 (23), 10346-10356.

Hama, H., Kurokawa, H., Kawano, H., Ando, R., Shimogori, T., Noda, H., Fukami, K. Sakaue-Sawano, A., Miyawaki, A., 2011. Scale: a chemical approach for fluorescence imaging and reconstruction of transparent mouse brain. Nat. Neurosci. 14 (11), $1481-1488$

Helmstaedter, M., Briggman, K.L., Turaga, S.C., Jain, V., Seung, H.S., Denk, W., 2013. Connectomic reconstruction of the inner plexiform layer in the mouse retina. Nature 500 (7461), 168-174.

Hussman, J.P., Chung, R.H., Griswold, A.J., Jaworski, J.M., Salyakina, D., Ma, D., Konidari, I., Whitehead, P.L., Vance, J.M., Martin, E.R., Cuccaro, M.L., Gilbert, J.R., Haines, J.L., Pericak-Vance, M.A., 2011. A noise-reduction GWAS analysis implicates altered regulation of neurite outgrowth and guidance in autism. Mol. Autism. 2 (1), 1.

Jongbloets, B.C., Pasterkamp, R.J., 2014. Semaphorin signalling during development. Development 141 (17), 3292-3297.

Joshi, P.S., Molyneaux, B.J., Feng, L., Xie, X., Macklis, J.D., Gan, L., 2008. Bhlhb5 regulates the postmitotic acquisition of area identities in layers II-V of the developing neocortex. Neuron 60 (2), 258-272.
Kruger, R.P., Aurandt, J., Guan, K.L., 2005. Semaphorins command cells to move. Nat. Rev. Mol. Cell Biol. 6 (10), 789-800.

Kullander, K., Croll, S.D., Zimmer, M., Pan, L., McClain, J., Hughes, V., Zabski, S. DeChiara, T.M., Klein, R., Yancopoulos, G.D., Gale, N.W., 2001. Ephrin-B3 is the midline barrier that prevents corticospinal tract axons from recrossing, allowing for unilateral motor control. Genes Dev. 15 (7), 877-888.

Leyva-Diaz, E., Lopez-Bendito, G., 2013. In and out from the cortex: development of major forebrain connections. Neuroscience 254, 26-44.

Liu, Y., Shi, J., Lu, C.C., Wang, Z.B., Lyuksyutova, A.I., Song, X.J., Zou, Y., 2005. Rykmediated Wnt repulsion regulates posterior- directed growth of corticospinal tract. Nat. Neurosci. 8, 1151-1159.

Lopez-Bendito, G., Flames, N., Ma, L., Fouquet, C., Di Meglio, T., Chedotal, A., TessierLavigne, M., Marin, O., 2007. Robo1 and Robo2 cooperate to control the guidance of major axonal tracts in the mammalian forebrain. J. Neurosci. 27 (13), 3395-3407.

Metin, C., Deleglise, D., Serafini, T., Kennedy, T.E., Tessier-Lavigne, M., 1997. A role for netrin-1 in the guidance of cortical efferents. Development 124, 5063-5074.

Mitchell, K.J., 2007. The genetics of brain wiring: from molecule to mind. PLoS Biol. 5 (4), e113.

Mitchell, K.J., 2015. The genetic architecture of neurodevelopmental disorders. In: Mitchell, K.J. (Ed.), The Genetics of Neurodevelopmental Disorders. Wiley, pp. 1-28.

Mori, M., Kose, A., Tsujino, T., Tanaka, C., 1990. Immunocytochemical localization of protein kinase $\mathrm{C}$ subspecies in the rat spinal cord: light and electron microscopic study. J. Comp. Neurol. 299 (2), 167-177.

Okada, T., Keino-Masu, K., Suto, F., Mitchell, K.J., Masu, M., 2019. Data for 3D reconstruction of the corticospinal tract in the wild-type and Semaphorin 6A knockout adult brain. Data in Brief in press.

Paixao, S., Balijepalli, A., Serradj, N., Niu, J., Luo, W., Martin, J.H., Klein, R., 2013. EphrinB3/EphA4-mediated guidance of ascending and descending spinal tracts. Neuron 80 (6), 1407-1420.

Perez-Branguli, F., Zagar, Y., Shanley, D.K., Graef, I.A., Chédotal, A., Mitchell, K.J., 2016. Reverse signaling by Semaphorin-6A regulates cellular aggregation and neuronal morphology. PLoS ONE 11 (7), e0158686.

Renier, N., Wu, Z., Simon, D.J., Yang, J., Ariel, P., Tessier-Lavigne, M., 2014. iDISCO: a simple, rapid method to immunolabel large tissue samples for volume imaging. Cell 159 (4), 896-910.

Rolf, B., Bastmeyer, M., Schachner, M., Bartsch, U., 2002. Pathfinding errors of corticospinal axons in neural cell adhesion molecule-deficient mice. J. Neurosci. 22 (19), $8357-8362$.

Rünker, A.E., Little, G.E., Suto, F., Fujisawa, H., Mitchell, K.J., 2008. Semaphorin-6A controls guidance of corticospinal tract axons at multiple choice points. Neural Dev. 3,34 .

Rünker, A.E., O’Tuathaigh, C., Dunleavy, M., Morris, D.W., Little, G.E., Corvin, A.P., Gill, M., Henshall, D.C., Waddington, J.L., Mitchell, K.J., 2011. Mutation of Semaphorin 6A disrupts limbic and cortical connectivity and models neurodevelopmental psychopathology. PLoS ONE 6 (11), e26488.

Steward, O., Zheng, B., Ho, C., Anderson, K., Tessier-Lavigne, M., 2004. The dorsolateral corticospinal tract in mice: an alternative route for corticospinal input to caudal segments following dorsal column lesions. J. Comp. Neurol. 472 (4), 463-477.

Susaki, E.A., Tainaka, K., Perrin, D., Kishino, F., Tawara, T., Watanabe, T.M., Yokoyama, C., Onoe, H., Eguchi, M., Yamaguchi, S., Abe, T., Kiyonari, H., Shimizu, Y., Miyawaki, A., Yokota, H., Ueda, H.R., 2014. Whole-brain imaging with single-cell resolution using chemical cocktails and computational analysis. Cell 157 (3), 726-739.

Welniarz, Q., Dusart, I., Roze, E., 2016. The corticospinal tract: evolution, development, and human disorders. Dev. Neurobiol. 77 (7), 810-829. 